\title{
Die Österreichische Nationalbibliothek als Werkstätte slowenistischer Forschung
}

V prvem razdelku članka so predstavljeni slovenistični fondi v bivši dunajski Dvorni knjižnici in delo nekdanjih knjižničarjev iz slovenskih dežel (J. Kopitarja, F. Miklošiča, I. Prijatelja idr.), v drugem delu pa je prikazan delež slovenike v digitalni knjižnici. Poleg tega je obravnavana tudi nekdanja in današnja vloga Avstrijske narodne knjižnice kot prejemnice slovenističnih obveznih izvodov, v novejšem času še zlasti iz avstrijske Koroške, ter dejavnost $\mathrm{v}$ zvezi $\mathrm{z}$ vnašanjem slovenike $\mathrm{v}$ zahodnoevropsko podatkovno bazo in $\mathrm{z}$ digitalizacijo slovenskih besedil. Avtorica opozarja tudi na možne raziskovalne teme in deziderate ter na nujnost sodelovanja med znanstveniki in knjižničarji.

\section{VORBEMERKUNG}

Die Wiener Slowenistik ist in der glücklichen Lage, was Quellen, Materialien und den wissenschaftlichen Nachwuchs betrifft, aus dem Vollen schöpfen zu können. Trotz ihrer anhaltenden ungerechtfertigten Marginalisierung (Sturm-Schnabl 1997) stellt sie keineswegs ,eine einsame Wissenschaft innerhalb der [...] Slawistik“ dar (Gesemann 2000: 38), sondern ein höchst lebendiges und attraktives Forschungsgebiet. Diesem sollte als einer Inlandsphilologie die besondere Aufmerksamkeit der relevanten staatlichen Einrichtungen gelten.

Zunächst eine Begriffsklärung: Ich betreue in der Österreichischen Nationalbibliothek unter anderem das Fach Slowenistik. Für die Zwecke des bibliothekarischen Tagesgeschäftes definiere ich „Slowenistik“, abweichend von der universitären Sicht, als Gesamtheit sowohl der wissenschaftlichen als auch der populären Beschäftigung mit slowenienrelevanten Inhalten. Diese Auffassung greift also weit über die reine Philologie - Literatur- und Sprachwissenschaft - hinaus in kulturwissenschaftliche, historische, soziologische Bereiche und bezieht auch vorwissenschaftliche, rein praktische Informationsangebote ein. 


\section{BESTÄNDE UND BIBLIOTHEKARE}

In der Österreichischen Nationalbibliothek, der Erbin der Wiener kaiserlichen Hofbibliothek (Palatina Viennensis) mit einer bis ins späte Mittelalter zurückreichenden Tradition, hat sich im Lauf der Jahrhunderte ein umfangreicher Bestand an Publikationen, Dokumenten und Quellen aller Art angesammelt: Handschriften und Autographen, Bild- und Notenmaterial, Ansichten und Karten, Flugblätter, Plakate, Postkarten, Exlibris, Zeitungen und Zeitschriften - und natürlich Bücher. Die Gesamtheit dieser Objekte verleiht der Österreichischen Nationalbibliothek - der heute durchaus unerwünschten Konnotation ihres Namens zum Trotz! - den Charakter eines hybrid-nationalen oder eigentlich transnationalen Gedächtnisortes. „Slovenica“, in analoger Definition zur „Slowenistik“, finden sich in fast allen Bereichen des Hauses: in den Sammlungen alter ${ }^{1}$ und neuer Druckschriften ${ }^{2}$, der Handschriftensammlung $^{3}$, Musiksammlung, Kartensammlung, Esperantosammlung ${ }^{4}$, Flugschriftensammlung, ${ }^{5}$, und nicht zuletzt im Bildarchiv ${ }^{6}$, das die ehemalige habsburgische Familien-Fideikommiss-Bibliothek enthält.

Mit ihren k.k. Hofbibliotheks-Wurzeln reicht die ÖNB noch tief in das universalistische Weltbild des Barocks hinein. Gerade im Hinblick auf slawische Druckwerke stellt sich die Frage, was in der Wiener Palatina bewusst gesammelt wurde und was, oder besser: welcher Anteil an der Gesamtheit der Bestände mit slawischen Bezügen, sich gewissermaßen durch die Zentripetalkraft der Hofbibliothek als Jahrhunderte lang führende Wissenschaftsinstitution des Habsburgerreiches hier angelagert hat.

Wir alle kennen Kopitars freudige Äußerung in dem Zusatz zu seiner Grammatik der slavischen Sprache in Krain, Kärnten und Steyermark:

Kurz vor Beendigung dieser Blätter hat mich das günstige Schicksal nach Wien geführt. Wie froh überrascht war ich, hier auf der kaiserlichen Hofbibliothek beinahe den ganzen

${ }^{1}$ Die Sammlung von Inkunabeln, alten und wertvollen Drucken (SIAWD) enthält Druckschriften bis einschließlich 1850 samt der zugehörigen Fachliteratur sowie besonders seltene oder bibliophile Drucke ohne zeitliche Begrenzung. Der historische Druckschriftenbestand der ÖNB von 1500 bis 1929 ist über das Bibliothekssystem ALEPH in zwei Online-Katalogen recherchierbar: NB-Opac 1501-1929; die seit 1992 von der ÖNB erworbene Literatur mit den Erscheinungsjahren 1501-1929 findet sich im NB-Opac ab 1992. Weitere Recherchemöglichkeiten zum alten Druckschriftenbestand bieten zwei Spezialkataloge in einer Imageversion (KatZoom): der Druckortekatalog 1501-1800 und der Gattungskatalog 1501-1929. Für die Benützung der Bestände der SIAWD steht der Augustinerlesesaal (Haupteingang Josefsplatz) zur Verfügung.

2 Die Druckschriftensammlung, heute als Moderne Bibliothek bezeichnet, ist die umfangreichste Einheit der ÖNB, sie beherbergt Bücher, Zeitungen und Zeitschriften mit Erscheinungsjahr ab 1851, dazu diverse Mikroformen, Videos, CDs, CD-Roms, etc. aus allen Wissensgebieten mit Schwerpunkt auf den Humaniora, die Benützung erfolgt in den Lesesälen am Heldenplatz, wo auch die wiss. Informationsabteilungen zu finden sind.

3 Handschriften-, Autographen- und Nachlass-Sammlung.

4 Sammlung für Plansprachen und Esperantomuseum.

5 Flugblätter-, Plakate und Exlibris-Sammlung.

${ }^{6}$ Ehem. Bildarchiv, Porträtsammlung und Fideikommissbibliothek. 
Schatz unsrer Literatur aus der Truber'schen Periode, sammt den Glagolischen und Kyrillischen Nebenerzeugnissen derselben, beysammen zu finden. (Kopitar 1808: 388 f., Hervorhebung im Original)

Die Provenienz der ersten slowenischen gedruckten Bücher, bekanntlich Trubars unikaler Catechismus In der Windischenn Sprach aus dem Jahr 1550, mit dem Abecedarium und der klein Catechismus In der Windischen Sprach aus dem selben Jahr und der zweiten Version Abecedarivm. Ene Buqvice aus 1555 zusammengebunden, erscheint durch einen Brief aus dem Jahr 1560 belegt, der im Haus-Hof- und Staatsarchiv aufbewahrt wird. Trubar ersucht darin den König von Böhmen, Erzherzog Maximilian, um Prüfung der ,lutherischen Rechtgläubigkeit“ seiner Bücher, die er dem Schreiben beilegt.

Als der erste professionelle Bibliothekar der Hofbibliothek im Jahr 1575 die vorgefundenen Bücher katalogisiert, zählen bereits einige slowenische Drucke zur 7379 Positionen umfassenden Sammlung und damit zum sogenannten „Ältesten Bestand“ der Palatina.

Die in der Zeit der Gegenreformation und späterhin erworbenen slowenischen Frühdrucke lassen keine einheitliche Provenienz mehr erkennen. Da finden sich etwa Bestände aus ehemaligen Adelsbibliotheken (aus der Windhaag'schen Bibliothek mit schönem barockem Wappenexlibris der erste Teil des slowenischen Neuen Testamentes von Mandelc in Laibach) oder Bücher aus aufgelassenen Klöstern (wie das eine der zwei im Besitz der ÖNB befindlichen Exemplare der Dalmatin-Bibel, das aus dem Wiener Kloster der Unbeschuhten Augustiner stammt). Auch durch Geschenke, Nachlässe und Ankauf ganzer Sammlungen fanden noch einzelne ältere Slovenica in die Palatina. Einige Exemplare gelangten durch gezielte Ankäufe im 19. Jahrhundert in die Bibliothek. Dalmatins Übersetzung Jezus Sirach etwa wurde der Palatina von einem Praktikanten geschenkt, der in der Widmung vermerkt, Kopitar habe ihn auf das Buch aufmerksam gemacht. Bohorič' Arcticae horulae wurde von Kopitar aus dem Familienbesitz Václav Hankas angekauft, das Buch trägt das Exlibris „Z Hankových“.

Eine Provenienzgeschichte dieses wertvollen Bestandes, von dem jedes einzelne Exemplar ein Rarum, wenn nicht ein Rarissimum ist, steht noch aus. Gerade im Fall des slowenischen Schrifttums könnte das Wissen um die Vorbesitzer der frühen Drucke und um den Weg dieser Drucke in die Wiener Hofbibliothek als Beitrag zur nationalen Kulturgeschichte dienen.

Slovenica, Slavica überhaupt, wurden weder zu Zeiten der Palatina noch später in der Österreichischen Nationalbibliothek von den deutsch- und anderssprachigen Beständen getrennt erfasst und aufgestellt, und dies gilt bis heute. Eine Kennzeichnung der Bücher nach sprachlichen Kriterien - graece, latine, gallice, polonice, slovenice etc. - bestand nur in den alten Inventaren, sie ist heute noch im „Katalog 1501$1929^{67} \mathrm{zu}$ sehen. Im übrigen waren die Einteilungskriterien ab dem Zeitpunkt, als

\footnotetext{
${ }^{7}$ www.onb.ac.at / Kataloge.
} 
man welche anwendete, rein technischer Natur: Man trennte zunächst Drucke (Impressen) von Handschriften (Manuskripten) und diese wiederum von Kupferstichen, im 19. Jahrhundert kamen als eigene Gruppe die Musikalien und Noten dazu. Dieser Umstand erklärt, warum unser Haus - zum Nachteil der Benützer, aber mehr noch zum Leidwesen der Bibliothekare - keine eigene „Slavica“- oder „Osteuropa-Abteilung" besitzt, wie etwa die Staatsbibliothek zu Berlin oder die British Library (British Library Slavonic and East European Collections). Zur Sicherstellung der Osteuropa-Kompetenz des Hauses für die Zukunft konnte lediglich die Einrichtung einer „Arbeitsgruppe Slavica“ erreicht werden. Dies geschah im Jahr 2001, noch vor der Ausgliederung der Österreichischen Nationalbibliothek aus der unmittelbaren Bundesverwaltung. ${ }^{8}$ Diese „Arbeitsgruppe Slavica“ ist eine fragile Konstruktion, die keinen Abteilungsstatus besitzt und daher in der Bibliotheksorganisation nicht fest verankert ist. Ihr gehören heute vier Slawisten an, denen jedoch neben ihren slawistischen Agenden zunehmend anderweitige Referenten- bzw. organisatorische Funktionen übertragen werden.

Überlegungen in Richtung einer Verselbständigung der slawischen Bestände innerhalb der Bibliothek gab es schon im Jahr 1810: Noch vor seiner Anstellung in der Palatina setzte Kopitar (1810-1844) ${ }^{9}$ alle Hebel zum Ankauf der Slavica-Bibliothek aus dem Nachlass des Tschechisch-Professors Josef Zlobický durch die Hofbibliothek in Bewegung. So „bekäme die Hofbibliothek eine schöne Grundlage zu einer Slavischen Abtheilung", schreibt er an Josef Dobrovský in Prag (Dobrovský - Kopitar 1885: 129). Diese „Slavische Abtheilung“ hätte zunächst die mit slawistischem, slowenistischem Material reichbestückte wissenschaftliche Werkstatt zur Realisierung von Kopitars eigenen hochfliegenden austroslawischen Plänen sein sollen. Die Einrichtung einer derartigen Sondersammlung, herausgelöst aus der Druckschriftensammlung der Hofbibliothek, hatte jedoch keine Chance auf Realisierung, sie lief, könnte man polemisch sagen, dem „Geist des Hauses“ zuwider, der sich nicht für eine politische Sache instrumentalisieren ließ (Hüttl-Hubert 1994).

Den erstaunlich guten Ruf, den die Österreichische Nationalbibliothek bei Historikern und Philologen als Fundstätte tschechischer, slowakischer, polnischer, ukrainischer, kroatischer, serbischer und eben slowenischer Werke aus der ehemaligen Habsburgermonarchie genie $\beta t,{ }^{10}$ verdankt sie einem Instrument hoheitlicher Inter-

${ }^{8}$ Der derzeitige Status der ÖNB ist der einer „Wissenschaftlichen Anstalt öffentlichen Rechts", analog dem Status der Bundesmuseen, wie z. B. des Kunsthistorischen Museums.

$9 \mathrm{Im}$ Weiteren geben die Jahreszahlen in Klammer jeweils die Dienstzeit der genannten Mitarbeiter in der Hof- bzw. Nationalbibliothek an. Zu Kopitars Tätigkeit in der Palatina vgl. Hüttl-Hubert 1994. Eine kurzgefasste Darstellung der übrigen slowenischen Mitarbeiter der Hofbibliothek vgl. Hartmann 1973.

10 Dazu eine Stimme aus Slowenien: „Ker pa je dunajska ,nacionalka ena najbogatejših knjižnic, je lahko Simonič zajel skoraj vse, kar je bilo natisnjenega. Strokovnjaki ocenjujejo, da je v svoje delo zajel več kot $75 \%$ vseh slovenskih knjig od leta 1550 do leta 1900 “ (Lipnik 1999: 14). Franz Simonič, Bibliothekar an der Wiener Universitätsbibliothek 
vention, dem sogenannten „Pflichtexemplar“, das für reichlichen Zufluss an Literatur aus allen Kronländern sorgte (Strebl 1970). Es findet seinen Ursprung in den Druckerprivilegien und der Bücherzensur zu Anfang des 17. Jahrhunderts. Die Patente und Erlässe mussten mehrmals wiederholt und neu gefasst werden, was nicht für die Akzeptanz dieses Rechtsinstitutes bei den betroffenen Verlegern und selbst bei den mit der Durchführung befassten Behörden spricht. Trotz der rigide klingenden Vorschriften, trotz Strafandrohungen, scheint eine lückenlose Ablieferung aller Drucke zu keiner Zeit gelungen zu sein.

Machen wir die Probe aufs Exempel: Aus der Zeit der „nationalen Wiedergeburt" ist Pohlins Kraynska gramatika in der ersten und zweiten Auflage vorhanden. Sein Dictionarium Slavicum Carniolicum fehlt. Linharts Versuch einer Geschichte von Krain ist vorhanden, auch die Blumen aus Krain, die Erstausgabe seiner Županova Micka oder des Matiček suchen wir vergeblich. Von Valentin Vodnik ist erwartungsgemäß die antinapoleonische Collin-Übersetzung Pesmi za brambovce, aber auch die Pismenost ali gramatika za perve šole vorhanden, die von ihm redigierte erste slowenische Zeitung, Lublanske novice, und die Kalenderdrucke fehlen.

Nach dem Ende des Römisch-deutschen Kaiserreichs musste die Pflichtablieferung für die habsburgischen Länder neu geregelt werden. 1807 wurden den Universitäts- und Lizealbibliotheken das Pflichtexemplarrecht zugestanden - seit damals erhielt die Licejska knjižnica in Laibach, die Vorgängerin der Narodna in univerzitetna knjižnica (NUK), ein Exemplar aller im Land Krain gedruckten Bücher, wodurch sie den Charakter einer Landesbibliothek gewann. 1808 wurde dann auch die Abgabe eines kostenlosen Exemplars aller in den k.k. Ländern neu aufgelegten oder nachgedruckten Werke, Kupferstiche und Landkarten ,zum Gebrauch der k.k. Hofbibliothek als einer gemeinnützigen Anstalt" dekretiert (Handbuch 2003: 45). Ab 1811 sollten die Bücherrevisionsämter, die bei den Polizeistellen der Landeshauptstädte zum Zweck der Zensur eingerichtet waren, die Druckwerke an die Palatina weiterleiten. (Dadurch erübrigte sich ein direkter Kontakt der Bibliothek mit den Verlegern.) Diese Regelung galt bis zur Aufhebung der Bücherrevisionsämter im Jahr 1848, die ein Stocken der Ablieferung bewirkte und in der Folge empfindliche Lücken in den Bibliotheksbeständen hinterließ. So fehlen zum Beispiel die Jahrgänge 1850-52 des Laibacher Tagblattes und die Jahrgänge 1849-51 der Kmetijske in rokodelske novice bis heute in den Beständen der ÖNB.

Die slowenischen Bestände der Bibliothek, und nicht nur diese, erweisen sich bei der Überprüfung aufgrund retrospektiver Bibliographien also als weitaus lückenhafter als angenommen. Dies hat zweierlei Ursachen:

Zum einen war man ab dem Vormärz in den aufstrebenden nationalen Zentren aus naheliegenden Gründen zurückhaltend mit Informationen über die lokale Buchproduktion. Ein anderer, weit gewichtigerer Grund für die seit dem Einsatz der elek-

(1877-1906), hat seine retrospektive „Slovenska bibliografija“ (T.1. 1903-1905) vorwiegend auf autoptischer Grundlage erarbeitet. 
tronischen Kataloge immer klarer zutage tretenden Bestandslücken liegt in dem in der Zwischenzeit radikal veränderten Sammelverhalten der Bibliothek. Heute werden die inländischen Druckschriften in der Nationalbibliothek völlig wertfrei und in größtmöglicher Vollständigkeit gesammelt und archiviert, noch weit in das 19. Jahrhundert hinein wurden die ordnungsgemäß abgelieferten Pflichtexemplare von den Hofbibliothekaren einer strengen Prüfung unterzogen und Druckwerke ausgeschieden, die ,, unter allen Umständen und in jeder Hinsicht wertlos sind " (Akten: HB 22/1853). So wurden damals unzählige Bücher und Broschüren, insbesondere solche populärer Art, die heute etwa als sozialhistorisches und kulturwissenschaftliches Quellenmaterial von Interesse wären, von unseren Amtsvorgängern ganz nach Vorschrift auf dem Tauschweg oder gar über den Altpapierhandel entsorgt.

Die aus heutiger Sicht logische Erwartung, die Anwesenheit eines slowenischen Bibliothekars im Personalstand der Hofbibliothek garantiere das Vorhandensein der zeitgenössischen slowenischen Literaturproduktion in deren Beständen, geht also ins Leere. Kopitar hatte vorwiegend mit Handschriften und alten Drucken zu tun, also mit materiell und ideell wertvollen Objekten. Kleinere Literaturgattungen, populäre Publikationen, literarische Zeitdokumente blieben weitgehend am Rande seiner Wahrnehmung, und für die Behandlung der Pflichtexemplare war er nie zuständig.

Der Autor der oben zitierten Instruktion ist übrigens niemand Geringerer als Franz Miklosich (1844-1862), Kopitars Nachfolger als Zensor und Bibliothekar. Man kann davon ausgehen, dass Miklosich mit der erstaunlich scharfen Formulierung ,unter allen Umständen und in jeder Hinsicht wertlos " nicht seine persönliche Meinung über die Wertigkeit von Büchern zum Ausdruck bringen wollte, sondern dass er damit lediglich - als Philologe und Jurist - dem dienstlichen Auftrag entsprach, eine lang geübte bibliothekarische Praxis in eine verbindliche Form zu bringen.

Vor einiger Zeit ist als eines der allerletzten Stücke aus Miklosich' Nachlass ein Exemplar des von Janez Bleiweis bei Blaznik in Ljubljana herausgegebenen Koledarčik slovenski za navadno leto 1854 in die Bibliothek gelangt. Der Kalender enthält Miklosich' jugendliches Porträt als Frontispiz, im Anhang die Lebensbilder von Valentin Vodnik, ebenfalls mit einem Porträt, und Franz Miklosich. Darin schreibt Ivan Navratil:

[...] slavni naš Kopitar [...] ga pregovori leta 1844, da pravdaríjo popustí in v pridvorno knjižnico v službo stopi, v kteri je obilno pomočkov za vsako znanstvo. $V$ tem veličanskem znanstvenem svetišču je začel bistroglavi Miklošić [!] nemudoma učene bukve marljivo prebirati, staroslovenske rokopise pregledavati in zlasti slovanske jezike premišljávati. Neprenehoma je delal - bral, prevdarjal in bukve pisal. - Tukaj se je slava njegova počela. (Koledarčik 1853: 38)

Hier erscheint die Hofbibliothek nur als wissenschaftliches Schlaraffenland für einen hellen Kopf - dass sich der Held der Erzählung bei seinem Eintritt in die Palatina bereit erklären musste, „für die Beschreibung der Bücher und zur Bedienung des Lesepublikums“ (Geschichte 1968: 452) zur Verfügung zu stehen, bevor er sich den slawischen Handschriften widmen konnte, bleibt in diesem Kontext natürlich unerwähnt. Die Stelle erscheint als ins Populäre gekehrtes Lessing'sches Dictum 
„ich darf mich rühmen, dass ich mehr die Bibliothek als die Bibliothek mich benützen sollte“" (Barth 1996).

Bleiweis' Koledarčik illustriert anschaulich die Erwerbungspraxis in der Mitte des 19. Jahrhunderts, als ein weitverbreitetes Druckwerk, das eine praktische, volkspädagogische Funktion ausübte, noch als ,,in jeder Hinsicht wertlos“ und daher unwürdig für die Aufnahme in die kaiserliche Hofbibliothek galt - und ist übrigens auch ein Beweisstück dafür, dass Miklosich von den strengen dienstlichen Grundsätzen auch in eigener Sache nicht abwich.

Ein Erlass des Unterrichtsministeriums aus dem Jahr 1857 zeigt, dass man sich außerhalb der Hofbibliothek der Problematik allzu restriktiver Erwerbungen sehr wohl bewusst war:

Die [...] Bibliotheken haben ganz besonders jene Druckschriften zu sammeln und geordnet aufzubewahren, welche politische und Kirchengeschichte, die gesamte geistige Bewegung im Gebiet der Kunst, der Wissenschaft und des geselligen Lebens, die Natur und die Erzeugnisse des Landes [...] behandeln. Es ist hierbei stets im Auge zu behalten, daß solche Schriften für die historische Beurtheilung der Zeit, aus welcher sie stammen, in späterer Zeit von großem Wert sein werden. Solche Schriften bilden einen charakteristischen Bestandteil der bibliotheca patria. (Strebl 1970: 354)

In diesen Bahnen und schon mit dem Blick auf eine eigenstaatliche Zukunft bewegte sich dann die Erwerbungs- und Referententätigkeit von Ivan Prijatelj (19051919) und France Kidrič (1908-1920). Während deren Mitarbeit in der Palatina erreichten die Slovenica die größte Bestandsdichte und -tiefe der Zeit vor dem Ersten Weltkrieg. Prijatelj sorgte nicht nur für die Abgabe von Belletristik-Monographien sämtliche Erstausgaben seiner Freunde Cankar und Župančič -, auch zahlreiche Periodica und andere Publikationsformen sind aus dieser Zeit vorhanden. Hier hat man zum ersten Mal den Eindruck, dass zeitgenössische slowenische Drucke bewusst gesammelt und in der Hofbibliothek aufgestellt wurden. Jetzt scheute man sich nicht mehr, der Bibliothek fortschrittliches und kontroverses Material einzuverleiben. Als Beispiel sei die sozialdemokratische Zeitschrift Rdeči prapor mit dem zensurierten Cankar-Artikel ,Krvavi dnevi v Ljubljani“" vom September 1908 genannt.

Kopitar, Miklosich, Prijatelj, Kidrič, und später Hafner - jeder dieser hervorragenden Wissenschaftler hat seinen Beitrag zur quantitativen und qualitativen Exzellenz der Bibliothek geleistet und sich nebenher des reichhaltigen Angebotes der Palatina auf seine Weise und für seine persönlichen Zwecke bedient. So ist die Entstehung von Prijateljs umfangreichem literaturwissenschaftlichem, publizistischem, übersetzerischem und essayistischem Oeuvre auf dem Gebiet des Slowenischen (teilweise auch des Polnischen und Russischen) jedenfalls auch vor dem Background der Fonds der Hofbibliothek zu sehen. Die Frage, ob seine außerdienstlichen Aktivitäten, die ins Kulturpolitische hineinreichten, und ihm später den Ehrentitel eines ,narodni ideolog“ (Barbarič 1975: 14) eingebracht haben, vom Dienstgeber gebilligt, hingenommen, oder überhaupt wahrgenommen wurden, ist heute schwer 
zu beantworten. Wenn Prijatelj - nota bene als Angehöriger des habsburgischen Hofstaates ${ }^{11}$ - während des Krieges, aufgrund der in der Hofbibliothek einlaufenden Periodica, slowenische und tschechische Oppositionspolitiker mit wertvollen politischen Informationen versorgte, wie Anton Slodnjak mitteilt (Slodnjak 1975: 48), wirft das ein Licht auf die inneren Auflösungsvorgänge der zu Ende gehenden Donaumonarchie.

Nach 1918, der Abtrennung des slowenischen Kernlandes und dem Verlust der slowenischen Bibliothekare, geht die Anzahl der Slovenica, wie der Slavica überhaupt, im Bestand der Bibliothek erwartungsgemäß deutlich zurück. Es ist jedoch bemerkenswert, dass die Bibliotheksverantwortlichen sich in den Jahren der Rezession ihrer historischen Verantwortung gegenüber der Sammlung und ihren Benützern bewusst bleiben. Aus dem Jahr 1920 stammt eine programmatische Erklärung unter dem Titel „Die weltpolitische Bedeutung der Nationalbibliothek“, die ein klares Bekenntnis zur weiteren Pflege der slawischen Agenden und Bestände enthält (Geschichte 1968: 635 f.).

In den finanziell besonders schwierigen Zwischenkriegsjahren konnte die Nationalbibliothek eine über die Slowenistik hinaus sehr bedeutende Erwerbung verzeichnen: Miklosich' Sohn Moriz schenkte der Bibliothek die gesamte Korrespondenz seines Vaters, mehr als 3000 Briefe, das Zeugnis von Miklosich' weltweiten wissenschaftlichen Kontakten mit an die 900 Korrespondenzpartnern. Dieses Geschenk enthält auch den größten Teil der erhaltenen Kopitar-Briefe (Akten: HB 193/1924).

Unter einem völlig anderen Aspekt als je zuvor wurde das Sammeln von Büchern aus den slawischen Ländern in der NS-Zeit betrieben (Geschichte 1973: 128; Hall - Köstner 2006: 354-358). Die Nationalbibliothek wurde nun sieben Jahre lang ihrem Namen gerecht. Der aus Maribor/Marburg stammende Generaldirektor, SSObersturmbannführer Paul Heigl war klarerweise besonders am Schrifttum der versprengt in den südosteuropäischen Ländern lebenden deutschen Volksgruppen interessiert. Darüber hinaus setzte er sich dafür ein, die reichlich vorhandenen Slavica der Nationalbibliothek für Kriegszwecke nutzbar zu machen.

Heigl richtete in der Bibliothek im Jahr 1939 eine „Orient-Südosteuropa-Abteilung“ ein. Diese in der Nachfolgeinstitution der habsburgischen Hofbibliothek aus historischen und inhaltlichen Gründen absolut widersinnige Zusammenführung von slawischen mit hebräischen, orientalischen, chinesischen und japanischen Beständen wurde nach dem Krieg sang- und klanglos aufgelassen. Der Sammlung, zu deren Leiter ein Germanist [!] bestellt wurde, war unter anderem aufgetragen, das gesamte in der Bibliothek auffindbare Material aus dem südosteuropäischen Raum durch Spezialkataloge zu erschließen. Ein ehrgeiziges Vorhaben für eine Abteilung, der bis 1942 kein einziger Slawist angehörte! Eines der ersten Ziele war die Erstellung einer

${ }^{11}$ Die Mitarbeiter waren auch noch zu jener Zeit keine Staatsbeamten, sondern, wenn auch nur nominell, Angehörige des Hofes. 
Bibliographie der Wiener Drucke in den südosteuropäischen Sprachen, zu deren Publikation es kriegsbedingt leider nicht mehr gekommen ist. Das einzige konkrete Lebenszeichen der kuriosen Abteilung war eine 1942 im Prunksaal unter dem lapidaren Titel „Südosteuropa“ gezeigte Ausstellung. Dem kommentarlosen, hektographierten Katalog (Südosteuropa 1942) zu dieser Schau ist zu entnehmen, dass aus dem reichen Fundus slawischer Bestände - Handschriften, Autographen, Drucke, Ansichten und Karten - auch einige Slovenica gezeigt wurden. Unter anderem Briefe und Autographe von Kopitar, dessen Glagolita Clozianus, einige Miklosich-Ausgaben, einige Trubar-Erstausgaben.

Die in der Zeit zwischen 1938 und 1945 erschienenen wichtigen slowenischen Publikationen (beispielsweise Prijateljs fünfbändige Kulturna in politična zgodovina Slovencev 1848-1895, Edvard Kardeljs unter dem Pseudonym Sperans erschienene gesellschafts- und kulturpolitische Analyse Razvoj slovenskega narodnega vprašanja oder die von Rajko Nahtigal besorgte zweibändige Kopitar-Werkausgabe) sind sämtlich erst nach dem Krieg, während der Dienstzeit von Stanislaus Hafner (19521963), durch Kauf oder Tausch in die Bibliothek gelangt. Hafner selbst hat die Slovenica der Österreichischen Nationalbibliothek unter anderem für seine Studien über verschiedene Aspekte der kulturpolitischen Bedeutung Wiens im 19. Jahrhundert konsultiert. Mit ihm endete vorerst die Reihe der slowenischen Mitarbeiter, die es verstanden haben, dem bibliothekarischen ,aliis inserviendo consumor“ gerecht zu werden und zugleich in selbstbewusster Weise das Haus mit seinen Beständen und seiner Infrastruktur als Werkstätte für ihre eigenen wissenschaftlichen Arbeiten zu nutzen.

\section{SLOVENICA IN DER DIGITALEN BIBLIOTHEK}

Die Erwerbung von Literatur aus Slowenien und zu slowenienrelevanten Themen ist jedoch nach Hafners Abgang an die Universität Graz nicht abgebrochen, im Gegenteil: Vergleicht man die Anzahl der in den drei elektronischen Katalogen der Österreichischen Nationalbibliothek (1501-1929, 1930-1991, 1992-laufend) verzeichneten slowenischen Publikationen, kommt man zum - auf den ersten Blick - überraschenden Ergebnis, dass in diesen unterschiedlich langen Zeitabschnitten (430, 61, 15 Jahre) annähernd gleich viele Slovenica erworben wurden.

Erworben wurde und wird neben der gehobenen Belletristik samt deren Sekundärliteratur das ganze Spektrum der humanwissenschaftlichen Buchproduktion, alles selbstverständlich in Auswahl und mit besonderer Berücksichtigung von Publikationen zum gemeinsamen kulturellen Erbe sowie der für die Sondersammlungen der Bibliothek benötigten Referenzwerke. Erworben wird durch Kauf, Tausch und Geschenk (einschließlich der Belegexemplare), wobei den Tauschbeziehungen ${ }^{12}$ mit

12 Seit 2004 werden von den Verlagen nur mehr 2 Exemplare pro Buch an die ÖNB abgeliefert, die meist beide in die Bestände der Bibliothek aufgenommen werden, sodass für 
der Slowenischen Nationalbibliothek (NUK), der Universitätsbibliothek Maribor (UKM) und der Akademiebibliothek (SAZU) besondere Bedeutung zukommt. Geleitet von der Einsicht, dass ein kleines Land gezielte Anstrengungen unternehmen müsse, um seine kulturellen Leistungen international zur Geltung zu bringen, wurde bereits im Jahr 1982 im slowenischen Bibliotheksgesetz als eine der Aufgaben der Slowenischen Nationalbibliothek die Zuständigkeit für die Präsenz von Slovenica im Ausland festgeschrieben (Šetinc 2006: 9 f.). Dieser Passus wurde auch in die Neufassung des Gesetzes im Jahr 2001 übernommen und erleichtert die Versorgung der ausländischen Partnerbibliotheken mit slowenischer Literatur.

Die in Österreich verlegten oder gedruckten Slovenica gelangen nach wie vor durch die Institution des Pflichtexemplars in die Bibliothek. Eine aus dem Mediengesetz 1981 in das Bundesmuseen-Gesetz 2002 übernommene Bestimmung über die Ablieferung und Anbietung von Bibliotheksstücken ${ }^{13}$ soll die lückenlose Präsenz des inländischen Schrifttums in gedruckter und elektronischer Form in der Nationalbibliothek sicherstellen. So findet der Benützer die komplette Produktion der literarisch besonders aktiven und grenzüberschreitend publizierenden Kärntner und neuerdings der steirischen Slowenen an einer Stelle vereint und in der amtlichen, von der Nationalbibliothek seit 1946 laufend herausgegebenen Österreichischen Bibliographie verzeichnet. ${ }^{14} \mathrm{Ab} 2003$ wird dieses Verzeichnis nur mehr in elektronischer Form erstellt und ist über die Homepage der Österreichischen Nationalbibliothek abrufbar. $^{15}$

Gerade im Hinblick auf die Kärntner Slowenen erweist sich das Pflichtexemplar als nützliche Einrichtung: Zeitungen und Zeitschriften, Kalender, Pfarrblätter, Gemeindeinformationsschriften, Ausstellungskataloge, Schulschriften etc., ephemere Publikationsformen dokumentieren in ihrer Gesamtheit die gesellschaftspolitische Entwicklung und Befindlichkeit der Volksgruppe. Freilich liegt es auch an den Produzenten dieser Druckwerke, sie dorthin abzuliefern, wo sie formal und inhaltlich erschlossen, langzeitig archiviert und der Benützung zugänglich gemacht werden. Leider waren und sind die gesetzlichen Vorschriften manchen Produzenten kleinerer Drucke nicht bekannt, und so kommt es, dass gerade interessante Frühwerke, Studentenzeitungen und dergleichen, nicht vollständig in den Bibliotheken zu finden sind.

den Tausch nur mehr die sog. „,geförderten Publikationen“, die in größerer Zahl abgeliefert werden, zur Verfügung stehen. Dies belastet österreichischerseits die traditionell guten Tauschbeziehungen. Hinzu kommt noch, dass mit den wenigen freien Tauschexemplaren vorrangig „Auslandsaustriaca“ erworben werden sollen, da die zentrale Aufgabe der Österreichischen Nationalbibliothek das Sammeln von österreichrelevantem Schrifttum ist.

13 www.onb.ac.at/about/index.htm bzw. suchbar über die ÖNB-Homepage www.onb.ac.at

14 Gemäß den Usancen der nationalen Literaturdokumentation werden die in Österreich erschienenen „Auslandsslovenica“ auch sämtlich von der slowenischen Nationalbibliothek (NUK) gesammelt und im slowenischen Verbundkatalog (COBISS) verzeichnet.

5 www.bibliographie.onb.ac.at/biblio/ 
Die in Österreich produzierten und via Pflichtexemplar in die Bibliothek gelangten Slovenica und slowenienrelevanten Publikationen werden außer in die Österreichische Bibliographie und damit in den Verbundkatalog ALEPH auch in die Osteuropa-Datenbank EBSEES - Europäische Bibliographie zur Osteuropaforschung aufgenommen. Dies hat den doppelten Vorteil, dass einerseits durch die durchgängig autoptische Erfassung eine hohe Datenqualität gewährleistet ist, und andererseits der Benützer in der Österreichischen Nationalbibliothek den direkten Zugriff auf die Bestände hat. Erfasst werden in österreichischen Zeitschriften, Monographien, Festund Kongressschriften enthaltene osteuroparelevante Texte zu human- und sozialwissenschaftlichen Themen. Ab dem Jahrgang 1991 erscheint die Bibliographie in einer Datenbankversion, die im Internet zur Verfügung steht und auch über die Homepage der Österreichischen Nationalbibliothek erreichbar ist. ${ }^{16}$

Eine im Jahr 2004 mit slowenischen Fachkollegen initiierte Kooperation betrifft die Digitalisierung von Zeitschriften und Zeitungen im Rahmen des ,,virtuellen Zeitungslesesaales “ der Österreichischen Nationalbibliothek, ANNO. ${ }^{17}$ Zunächst ist daran gedacht, die Laibacher Zeitung (1783-1918) komplett in einer Image-Version ins Netz zu bringen. Dieses in der Tradition der Ordinari-Zeitungen stehende amtliche Organ mit seinen Beilagen Intelligenzblatt und Illyrisches Blatt hat die kulturelle Entwicklung des slowenischen Raumes sowohl dokumentiert als auch aktiv mitgestaltet - man denke nur an Prešerens bewegte Publikationsgeschichte mit dieser Zeitung. Die geplante Digitalisierung könnte die virtuelle Komplettierung dieses langlebigen Periodikums ermöglichen, dessen komplizierter Erscheinungsverlauf zu empfindlichen Bestandslücken in den Bibliotheken geführt hat. Und nicht zuletzt kommt ein konservatorischer Grund zum Tragen: Einzig die elektronische Verfügbarkeit des gesamten Textes in seiner ursprünglichen Erscheinungsform vermag das wertvolle Bibliotheksexemplar wirksam vor Verschleiß zu bewahren. Für dieses Vorhaben benötigen wir als Partner die Kollegen in der Slowenischen Nationalbibliothek (NUK) und deren Zeitungsbestand. Die zunächst angestrebte Imageversion, die es gestattet, in der Zeitung zu blättern, sie zu lesen und auszudrucken, kann als Vorarbeit für eine in der Zukunft zu erstellende, nach verschiedenen Kriterien textlich durchsuchbare Volltextversion gelten.

Ein Slovenicum, das von der Österreichischen Nationalbibliothek demnächst digitalisiert werden wird, ist das Landesgesetzblatt von Krain, Deželni zakonik in vladni list za kranjsko kronovino. Der komplette Text sämtlicher Ausgaben von 18491918 in slowenischer und deutscher Sprache wird voraussichtlich im Lauf des Jahres 2007 im „digitalen Lesesaal der Österreichischen Nationalbibliothek für Gesetze“, ALEX, abrufbar sein. ${ }^{18}$ In ALEX finden sich übrigens auch die in allen Teilen digi-

${ }_{17}^{16}$ www1.msh-paris.fr/betuee/ bzw. über die ÖNB-Homepage.

17 AustriaN Newspapers Online, erreichbar über http://anno.onb.ac.at/ bzw. über die ÖNBHomepage.

18 Alex - historische Rechts- und Gesetzestexte Online, erreichbar über http://alex.onb.ac.at bzw. über die ÖNB-Homepage. 
talisierten Stenographischen Protokolle des österreichischen Abgeordnetenhauses und des Herrenhauses für den kompletten Zeitraum 1861-1918. Ein anderer kleiner, bereits digitalisierter, Bestand mit Slowenienbezug findet sich im Digitalen ArchivRevolution 1848, wo sämtliche in der ÖNB befindliche Flugschriften aus dem Land Krain gelesen werden können. ${ }^{19}$

Wir sind eben dabei, eine Liste weiterer Textcorpora zu erstellen, die in den nächsten fünf Jahren digitalisiert werden sollen: historische Zeitungen und Zeitschriften, sowie historische Rechts- und Gesetzestexte. Sollten außer der genannten Laibacher Zeitung, die bereits auf der Liste steht, Wünsche oder Anregungen in diesem Zusammenhang auftauchen, darf ich um Kontaktaufnahme ersuchen.

Kürzlich hat sich die ÖNB bereit erklärt, ein weiteres slowenisches (und kroatisches) Digitalisierungsprogramm anzugehen. Konkret wird es zu einer Kooperation mit dem von Professor Prunč von der Universität Graz erarbeiteten und bei dieser Tagung vorgestellten Projekt Deutsch-slowenisch/kroatische Übersetzung 1848$1918^{20}$ kommen.

$\mathrm{Zu}$ welchen Themen, welchen Fragestellungen im Rahmen der Slowenistik kann die Österreichische Nationalbibliothek Wesentliches beitragen?

In der Österreichischen Nationalbibliothek steht der slowenistischen Forschung für Sprach- und Literaturwissenschaft ein zwar stellenweise unvollständiger, insgesamt aber repräsentativer Fundus an Primär- und Sekundärliteratur zur Verfügung. Darüber hinaus bietet unser Haus demjenigen optimale Werkstattbedingungen, der bereit ist, in die intellektuelle Umwelt und in das Alltagsleben der slowenischen Kulturschaffenden in der Zeit bis zum ersten Weltkrieg einzusteigen. Er findet hier zum Beispiel Prešerens oder Cankars Wiener Jahre in geistiger Hinsicht „,in situ“ vor. Er findet hier diejenigen Publikationen, die von den Slowenen rezipiert wurden, und jene, die die Fakten und Imponderabilien ihrer Lebenswelt, den verborgenen biographischen Subtext, bewahrt haben. Bücher, Zeitungen, Zeitschriften, in thematischer Verdichtung, zusammen mit dem vielfältigen Angebot der Spezialsammlungen an Non-book-material.

Ein Besuch der Österreichischen Nationalbibliothek ist gewissermaßen eine Einladung zur Interdisziplinarität, und diese sollte ohnehin ein Kompetenzkriterium der Slowenistik sein. Als Beispiel für interdisziplinäre Studien aufgrund des in der Bibliothek reichlich zur Verfügung stehenden Materials seien hier nur die im Anhang zu meinem Artikel über die Cankar-Jeglič-Kontroverse angeführten Anregungen bzw. Forschungsdesiderata genannt, insbesondere bezüglich der Untersuchung der durch Jeglič' Rote Broschüre ausgelösten, die Landesgrenzen von Krain weit überschrei-

${ }^{19}$ www.onb.ac.at / Sammlungen und Museen / Flugblätter-, Plakate- und Exlibris-Sammlung / Revolution 1848 / Geographische Systematik.

20 Vgl. Kurzbeschreibung (Einzelprojekte P17465) in der Projektdatenbank des Wissenschaftsfonds: www.fwf.ac.at 
tenden Pressekampagne sowie des Oeuvres des zweisprachig in weltanschaulich divergierenden Blättern publizierenden Franjo Pirc (Hüttl-Hubert 2005: 100).

Ein idealtypischer Benützer der Österreichischen Nationalbibliothek in unserem Sinne wäre ein Angehöriger der slowenischen Volksgruppe in Kärnten. Als doppelt kompetente Sprecher frei von Berührungsängsten mit der deutschen Sprache und dem österreichischen kulturellen Erbe, in das einzutreten die „matični Slovenci“ aus mancherlei Gründen Überwindung kostet, sind gerade die Kärntner Slowenen befähigt, alle Angebote des Hauses für slowenistische und komparatistische Studien optimal zu nutzen. Hier wirkt sich die Mehrsprachigkeit, die von der EU als „klares Vorbild und natürliches Grundelement der europäischen Kultur ${ }^{\text {‘21 }}$ gefördert wird, bereits unübersehbar als Heimvorteil aus.

Die Frage, mit der der Kulturphilosoph Dean Komel seinen Artikel über die Suche nach der slowenischen Identität betitelt, „Kje je Slovenija?“ (Komel 2005), glaube ich, in aller Bescheidenheit beantworten zu können: „Tudi v Avstrijski narodni knjižnici je Slovenija!“

\section{SCHLUSS}

In der Vergangenheit war es meines Wissens seitens des Institutes für Slawistik der Universität Wien nicht üblich, mit Absolventen Kontakt zu halten und Vertreter nichtuniversitärer Institutionen, zumal Bibliothekare, zu wissenschaftlichen Veranstaltungen beizuziehen. Gerade deshalb gilt den Veranstaltern mein besonderer Dank für die Einladung zu diesem Symposium.

Wenn im Anhang zur rezenten Geschichte der österreichischen Slawistik lapidar erklärt wird, dass ,die an anderen wissenschaftlichen Institutionen, wie der Österreichischen Nationalbibliothek [...] betriebene Forschung sich weitgehend in eigenen Bahnen bewegt" (Miklas 2005: 56), bleibt damit ein wesentlicher Bereich unserer und meiner Arbeit unerwähnt. Nämlich insbesondere der Beitrag - in Form von Publikationen und Ausstellungen samt zugehöriger Öffentlichkeitsarbeit ${ }^{22}$ - zur Aufarbeitung der voruniversitären Wiener Slawistik, die sich, angeregt und verkörpert durch den Slowenen Bartholomäus (Jernej) Kopitar in unserem Hause ereignet hat.

Wir benötigen die Fachwissenschaftler als Mitschöpfer der Bibliotheksbestände, aber auch als kollegiale Rezipienten und Kritiker unserer eigenen Projekte. Ein sinnvolles Zusammenwirken ist für die Erfüllung der Aufgaben beider Bereiche geradezu eine Notwendigkeit. Wenn die Bibliotheken lediglich unter dem Aspekt ihrer Funktion als Zulieferbetrieb für die Produktion von Geisteskapital gesehen werden, können sie unter den heutigen und absehbaren zukünftigen Bedingungen ihre Aufgaben nicht mehr zur Zufriedenheit der Scientific Community erfüllen. Ohne das Interesse und die Kooperationsbereitschaft der Wissenschaftler trocknet ein Referat in ei-

\footnotetext{
21 www.europarl.europa.eu/news v. 9.11.2006.

22 Vgl. die Jahresberichte der ÖNB ab 1987, insbes. für die Jahre 1987 und 1994.
} 
ner großen Allgemeinbibliothek aus, in einem Haus, das viele Fächer betreuen muss, und in dem ein Mitarbeiter für mehrere Fächer verantwortlich ist.

Die Zeiten des humanistisch gebildeten Bibliothekars sind unwiderruflich vorbei. Auch in Bibliotheken mit akademischem Anspruch verliert die wissenschaftliche Vertiefung im Bestandsaufbau zusehends an Boden. Bedenken Sie, dass diese Tendenz in absehbarer Zeit auch den Verlust eines klassischen Berufsfeldes für Ihre Absolventinnen und Absolventen bedeutet. Ihr Vis-à-vis in der Bibliothek ist demnächst ein in betriebswirtschaftlichen Kategorien denkender Informationsvermittler mit Fachhochschulabschluss. Wenn es sich zufällig um eine Absolventin oder einen Absolventen des Slowenischen Gymnasiums in Klagenfurt handelt, haben wir Glück gehabt!

Wir stehen alle auf dünnem Eis - Sie in der Universität mit ihren besorgniserregenden Entwicklungen, und wir Bibliothekare in der schönen neuen Info-Welt im Jahr 606 nach Gutenberg, im Jahr 8 nach Google.

\section{Quellen- und Literaturverzeichnis}

Akten:

Barbarič 1975:

Barth 1996:

Dobrovský - Kopitar 188

Geschichte 1968:

Geschichte 1973:

Gesemann 2000:

Hafner 1985:

Akten der Hofbibliothek (HB), ab 1923 Akten der Nationalbibliothek (NB)

St. Barbarič, Srečanja in soočanja, in: Prijateljev zbornik ob stoletnici rojstva, Hg. St. Barbarič, Ljubljana, 5-17

R. Barth, Fürstenbibliotheken im 17. und 18. Jahrhundert, in: http://www.stub.unibe.ch/stub/vor196/07/lan.html

1885: Briefwechsel zwischen Dobrovský und Kopitar (1808-1828), Hg. Vatroslav Jagić, Berlin - Sanktpeterburg

Geschichte der Österreichischen Nationalbibliothek, Hg. J. Stummvoll, 1.: Die Hofbibliothek (1368-1922), Wien Geschichte der Österreichischen Nationalbibliothek, Hg. J. Stummvoll, 2.: Die Nationalbibliothek (1923-1967), Wien

G. Gesemann, Slovenistik - Literaturwissenschaft in Deutschland, Bulletin der deutschen Slavistik 6, 34-39

St. Hafner, Geschichte der österreichischen Slawistik, in: Beiträge zur Geschichte der Slawistik in nichtslawischen Ländern, Hg. J. Hamm, G. Wytrzens (= Schriften der Balkankommission 30), Wien, 11-88

Hafner - Miklas - Ertl 2005: St. Hafner - H. Miklas - E. Ertl, Materialien zur Geschichte der Slawistik in Österreich nach 1938, in: Beiträge zur Geschichte der Slawistik in den nichtslawischen Ländern, Hg. G. Brogi Bercoff, P. Gonneau, H. Miklas (= Schriften der Balkankommission $46=$ Institut d'Ėtudes Slaves, Traveaux 46), Wien, 27-87

Hall - Köstner 2006: M. Hall; Ch. Köstner, ,... allerlei für die Nationalbibliothek zu ergattern ...": eine österreichische Institution in der NS-Zeit, Wien

Handbuch 1994: $\quad$ Handbuch der historischen Buchbestände in Österreich, Hg. W. Buchinger, K. Mittendorfer, 1,1, Wien - Hildesheim

Hartmann 1973:

B. Hartmann, Die slowenischen Bibliothekare der Wiener Hofbibliothek, in: Österreich und die Südslawen (= Internationales Kulturhistorisches Symposion Mogersdorf 2), Eisenstadt, 99-110

Hüttl-Hubert 1994: $\quad$ E. Hüttl-Hubert, Bartholomäus Kopitar und die Wiener Hofbibliothek, in: Bartholomäus (Jernej) Kopitar: neue Studien und Materialien anläßlich seines 150. Todestages, Hg. W. Lukan, Österreichische Ost- 
hefte 36/3, Wien - Köln - Weimar, 521-588; zugl. Osthefte, Sonderband 11, 153-219

Hüttl-Hubert 2005: $\quad$ E. Hüttl-Hubert, Verhinderte Bestseller - gelungene Skandale. Ivan Cankars „Erotika“, Anton Bonaventura Jeglič” „Rote Broschüre“, in: Historische Bestseller, Hg. Ch. Gastgeber, Biblos 54/1, 79-103

Jahresbericht:

Katičić 1998:

Österreichische Nationalbibliothek. Jahresbericht, Wien, $1987 \mathrm{ff}$.

R. Katičić, Die Wiener Slawistik an der Schwelle zum dritten Jahrtausend, Vortrag, gehalten am 19.10.1998 anlässlich der Eröffnung des Universitätscampus, Typoskript

Koledarčik 1853: J. Bleiweis, Koledarčik slovenski za navadno leto 1854, Ljubljana

Komel 2005:

Kopitar 1808:

D. Komel, Kje je Slovenija?, Nova revija $25=282-283,1-14$

B. Kopitar, Grammatik der Slavischen Sprache in Krain, Kärnten und Steyermark, Laibach

Lipnik 1999:

Miklas 2003:

J. Lipnik, Prispevek slovenske Štajerske v zakladnico (srednje)evropske kulture, in: Simoničev zbornik: gradivo s simpozija v Ivanjkovcih, Hg. J. Lipnik (= Zora 10), Maribor, 8-15

H. Miklas, Zur Rolle der Wiener akademischen Institutionen in der Geschichte der Slavistik des 19. Jahrhunderts, in: Histoire de la slavistique: le rôle des institutions, Hg. A. Bernard (= Traveaux 44), Paris, $17-44$

Miklas 2005:

s. Hafner - Miklas - Ertl 2005

Raecke 1998:

J. Raecke, „Slovenistische Linguistik“ oder „Sprachwissenschaftliche Slovenistik" oder "Slovenistik - Sprachwissenschaft", Bulletin der deutschen Slavistik 4, 44-51

Šetinc 2006: $\quad$ L. Šetinc, Slovenska nacionalna knjižnica, in: Slovenska nacionalna knjižnica. Ob 60-letnici preimenovanja v Narodno in univerzitetno knjžnico, Hg. D. Balažič, Ljubljana, 5-12

Slodnjak 1975:

A. Slodnjak, Ivan Prijatelj med slovstvenimi zgodovinarji svoje dobe, in: Prijateljev zbornik ob stoletnici rojstva, Hg. Št. Barbarič, Ljubljana, 19-54

Südosteuropa 1942: Ausstellung in der Nationalbibliothek, Wien

Strebl 1970:

M. Strebl, Die Wiener Hofbibliothek und die Pflichtexemplare, in: Festschrift Josef Stummvoll, Hg. J. Mayerhöfer, 1 (= Museion, Veröffentlichungen der Österreichischen Nationalbibliothek, N. F., 2.4.), Wien, 348-356

Sturm-Schnabl 1997: K. Sturm-Schnabl, Slowenistik an der Universität Wien als europäischer Beitrag, in: Die Funktion der Slawistik im europäischen Bildungswesen: eine alternative Geschichte und Prognose, Hg. O. Kronsteiner (= Die slawischen Sprachen 55), 95-114; zugl.: Trans - Internet-Zeitschrift für Kulturwissenschaften, 1998.3 www.inst.at/trans $/ 3 \mathrm{Nr} / \mathrm{sturm} . \mathrm{htm}$

Thiel 1977: J. Thiel, Zur Geschichte des Pflichtexemplars im Kaisertum Österreich (1807-1827), Biblos 26/3, 290-296

\author{
Eva Hüttl-Hubert \\ Österreichische Nationalbibliothek \\ Arbeitsgruppe Slavica \\ Josefsplatz 1, A-1015 Wien \\ eva.huettl-hubert@onb.ac.at
}


